\title{
Valoración de recursos naturales y ganadería en la zona centro de Veracruz, México
}

\author{
Ana Lid del Ángel Pérez', José Alfredo Villagómez Cortés², \\ Martín A. Mendoza Briceño ${ }^{3}$ y Andrés Rebolledo Martínez ${ }^{4}$
}

\begin{abstract}
RESUMEN
Se estudió en Veracruz, La Antigua y Boca del Río, Veracruz, México, la disposición a pagar (DAP) por servicios ambientales generados en una zona ganadera. Se aplicó un cuestionario al azar estratificado a 199 personas de áreas rurales y urbanas, preguntando sobre temas sociodemográficos, naturaleza, recursos naturales, ambiente y ganadería. Usando valoración contingente, complementado con entrevistas y trabajo de campo antropológico, se encontró que $83,42 \%$ del público en la muestra está dispuesto a pagar por un incremento de calidad ambiental. Un $85,93 \%$ cree que el medio ideal sería que los ganaderos usen prácticas ambientalmente eficientes, y ser compensados por ello por parte del Estado, que según la mayoría $(84,42 \%)$, podría venir de mayor carga impositiva. En esta DAP, por la vía tributaria fueron significativas $(p<0,05)$ las influencias del nivel de ingresos, y la disposición a aceptar un incremento de la normatividad ambiental aplicable a actividades pecuarias, y reducción de actividades en terrenos ganaderos. Otros mecanismos de pago aceptables para los entrevistados fueron un pago directo y único, y el apoyo económico promoviendo el desarrollo de la ganadería bovina. De ahí que estos resultados lleven a suponer que la opinión pública apoya una variedad de mecanismos de pago. También se detectó que el público apoya la emisión de normas ambientales complementarias, así como una estricta aplicación de la legislación ganadera vigente.
\end{abstract}

PALABRAS CLAVE:

Disposición a pagar, ganadería, servicios ambientales, valoración contingente, Veracruz.

\begin{abstract}
Willingness to pay (WTP) for environmental services from a cattle ranching region encompasing Veracruz, La Antigua, and Boca del Río, Veracruz State, Mexico, is reported. A survey questionnaire, randomly applied to a stratified sample of 199 individuals, about regional demographic attributes, natural resources, wildlands condition, environmental quality and animal husbandry was administered. Contingent valuation techniques were applied, and supplemented with interviews and anthropologic field work. Results revealed that $83,42 \%$ of the sampled individuals have a positive WTP for an improved environment, and $84,42 \%$ of respondents will accept increases in taxation as the venue to that end; $85,93 \%$ of the public in the sample will rather see that cattle ranchers should be compensated by government for the extra cost of an environmentally conscious management. WTP through increased taxation has statistically significant influences $(p<0,05)$ from household income, severity of regulations, and restrictions to ranching practices. Direct payment resulted acceptable, as well as additional government regulations mandating best ranching practices. Hence, these results led to support that public opinion favors a variety of payment mechanisms. Approval of additional environmental legislation, as well as strict enforcement of existing cattle raising regulations was also noticed.
\end{abstract}

Campo Experimental Cotaxtla. CIRGOC INIFAP. Ocampo 234 desp 322. 91700 Veracruz Ver. México. aldap28@yahoo.com

2 Department of Animal Sciences. University of Florida. PO Box 110910. Gainesville, Florida 32611-0910. villagomez@animal.ufl.du

3 Departamento de Bosques Naturales. FIPRODEFO. Bruselas 626. Col. Moderna. 44190 Guadalajara, Jalisco, Méx. martinmendoza@yahoo.com

4 Campo Experimental Cotaxtla. CIRGOC INIFAP. Ocampo 234 desp. 322. 91700 Veracruz Ver. México. 
KEYWORDS:

willingness to pay, cattle ranching, environmental services, contingent valuation, Veracruz.

\section{INTRODUCCIÓN}

Veracruz es una entidad con gran riqueza biológica y uno de los principales estados ganaderos del país, no solo por el número de cabezas, sino también por la superficie que se dedica a esta actividad, aproximadamente $45 \%$ del total de 7,19 millones de hectáreas (SAGAR, 1999). El uso histórico del suelo de la planicie costera del Golfo de México, se reporta desde la época colonial, debido a que la región presentaba una abundancia natural de pastos y vegetación, áreas inundables utilizadas en el pastoreo durante la estación seca, dunas y planicies (Del Ángel, 1994). En la actualidad los sistemas de ganadería se sustentan en ecosistemas simples de praderas y desmonte e inducción selectiva de gramíneas (Siemens, 1998), con base en el pastoreo de grandes extensiones. Las praderas están compuestas preferentemente por especies exóticas, algunas dominantes y otras demandantes de insumos comerciales para prosperar (plaguicidas, fertilizantes, etc.). Lo anterior sienta las bases para la transformación de elementos estructurales del ecosistema y cambios en el paisaje natural, mismos que diversos estudios económicos señalan como poco rentables (Velázquez, 1998).

Los problemas de la ganadería veracruzana y la búsqueda de solución convergen en asuntos de alimentación, sanidad y reproducción, con mínima investigación sobre problemas ambientales, de tal manera que si los valores ambientales de un ecosistema derivan en una utilidad pública (Macmillan et al., 2002), el impacto ambiental de las actividades humanas es de índole social y el afectado final no es solo el ambiente (suelo, agua y biodiversidad), sino también la sociedad en su conjunto. Por este motivo, el sistema de pastoreo se aprecia como una estrategia económica individual, cuyos costos ambientales los paga la sociedad debido a que las estimaciones económicas no consideran costos ambientales sobre los recursos naturales (Kondratyev et al., 1997; Stevens, et al., 2000). Por otra parte, los daños y la disminución a la calidad del ambiente solo aparecen como problema cuando la sociedad lo percibe como tal, a partir de lo cual surge el interés en conocer el valor (Zhongwei et al., 2001) que se otorga al ambiente y a la naturaleza en circunstancias similares a las de la ganadería.

Desde que existen estudios ambientales ha sido evidente la preocupación por el uso del recurso tierra. Las contradicciones entre estado del conocimiento, disponibilidad de tecnología y la práctica de los sistemas de producción ganadera, muestran la necesidad de trabajar en investigación básica. Esta debe sentar las bases que permitan vincular a los sistemas productivos ganaderos con sus consecuencias en los recursos naturales que afectan, además del deterioro en el bienestar de los involucrados, con un costo social (Capalbo et al., 1989; Capalbo y Antle, 1989; Lewan y Soderqvist, 2002), que en términos contables aparece como un subsidio que la sociedad paga a la ganadería, y en especial reduciendo la calidad de vida de las generaciones futuras que se verán privadas de opciones de uso y consumo debido a la simplificación del ecosistema en el paisaje del agostadero. Aunque la escala masiva de estos cambios es históricamente reciente, desde la época de la política económica denominada "crecimiento con estabilidad" (mediados del siglo $X X$ ), los problemas ambientales se han agravado, y deben ser más preocupantes para las instituciones que 
formulan las políticas regionales. La manera en que se han formado enormes bloques de terrenos transformados ha contribuido a reducir y fragmentar la cubierta silvestre, y a agregar niveles de dificultad y riesgo al problema. Por otra parte, en México se han realizado pocos estudios sobre el pago por servicios ambientales en ámbitos ganaderos, específicamente bienestar percibido e impacto social de una actividad con valor y precio como bienes de uso y consumo general. Uno de los pocos estudios es el de Romo (2005), sobre valoración de la protección a la mariposa monarca, pero más a manera de ejemplo metodológico, notificando que las autoridades federales consideran que estas técnicas son aceptables, y no tanto por buscar respuestas al tema estudiado. Por otra parte, economistas y diversos autores han manifestado la necesidad de contar con mecanismos que permitan a los propietarios de tierras recibir una recompensa por administrar dichos servicios ambientales o aceptar los daños, lo cual alentaría su conservación (Nasi et al., 2002; Soderqvist, 2003). Sin embargo, para tal efecto, el pago por servicios ambientales debe ser igual o menor al valor otorgado por la sociedad, previa existencia de una disposición a pagar por un servicio.

Por lo anterior, el presente trabajo presenta la valoración que la sociedad de Veracruz central efectúa de ambientes ganaderos, ganadería y su disposición a pagar por una modificación de prácticas que mejoren la calidad de bienes y servicios ambientales que prestan tales ambientes y la actividad ganadera. Ello, en oposición a los costos o consecuencias resultantes sobre el patrimonio natural, los recursos naturales y el ambiente regional, como producto de la actividad ganadera practicada con los sistemas de producción vigentes. Para tal propósito, se desarrolló un estudio de caso en la Cuenca del Río San Juan - La
Antigua, en Veracruz central (Figura 1), comprendiendo los municipios de La Antigua, Veracruz y Boca del Río, como territorio donde se producen los servicios ambientales y la ganadería objeto de este estudio, y la población de estos municipios como consumidora de estos servicios.

\section{METODOLOGÍA}

El clima de la región de estudio es cálido subhúmedo $A w_{2}$, con precipitaciones promedio de $850 \mathrm{~mm}$ y régimen de lluvias en verano, estableciéndose la estación seca a partir de noviembre, que coincide con la época de nortes (rachas de vientos huracanados). La temperatura media oscila entre 26 y $28{ }^{\circ} \mathrm{C}$ (García, 1988). La cuenca presenta una morfología compuesta por áreas de inundación estacional, planicies y dunas. Por ello, los ganaderos movilizan el ganado estacionalmente, para pastorear en los terrenos bajos durante la estación seca, y en tierras altas en la lluviosa.

La cubierta original de esta zona está descrita como de sabana con bosquetes abiertos ocasionales en medio de pastizales (Hernández, 2001; Siemens, 1998). Está reportada por los primeros conquistadores españoles, la presencia de frondosos bosques de galería con arbolado de grandes dimensiones rodeando los cauces de agua. Adicionalmente, la zona costera se caracterizó por vegetación de dunas y médanos, detrás de las cuales aparecían lagunas someras con vegetación acuática y de pantano (Sarabia, 2004). Las lagunas mayores (Conchal y Mandinga) tenían márgenes de manglar. La vida silvestre nativa de estos ambientes primarios era rica en variedad y en especies útiles. Todo este paisaje se transformó con la llegada de la ganadería, iniciada por Hernán Cortés y sus capitanes, 


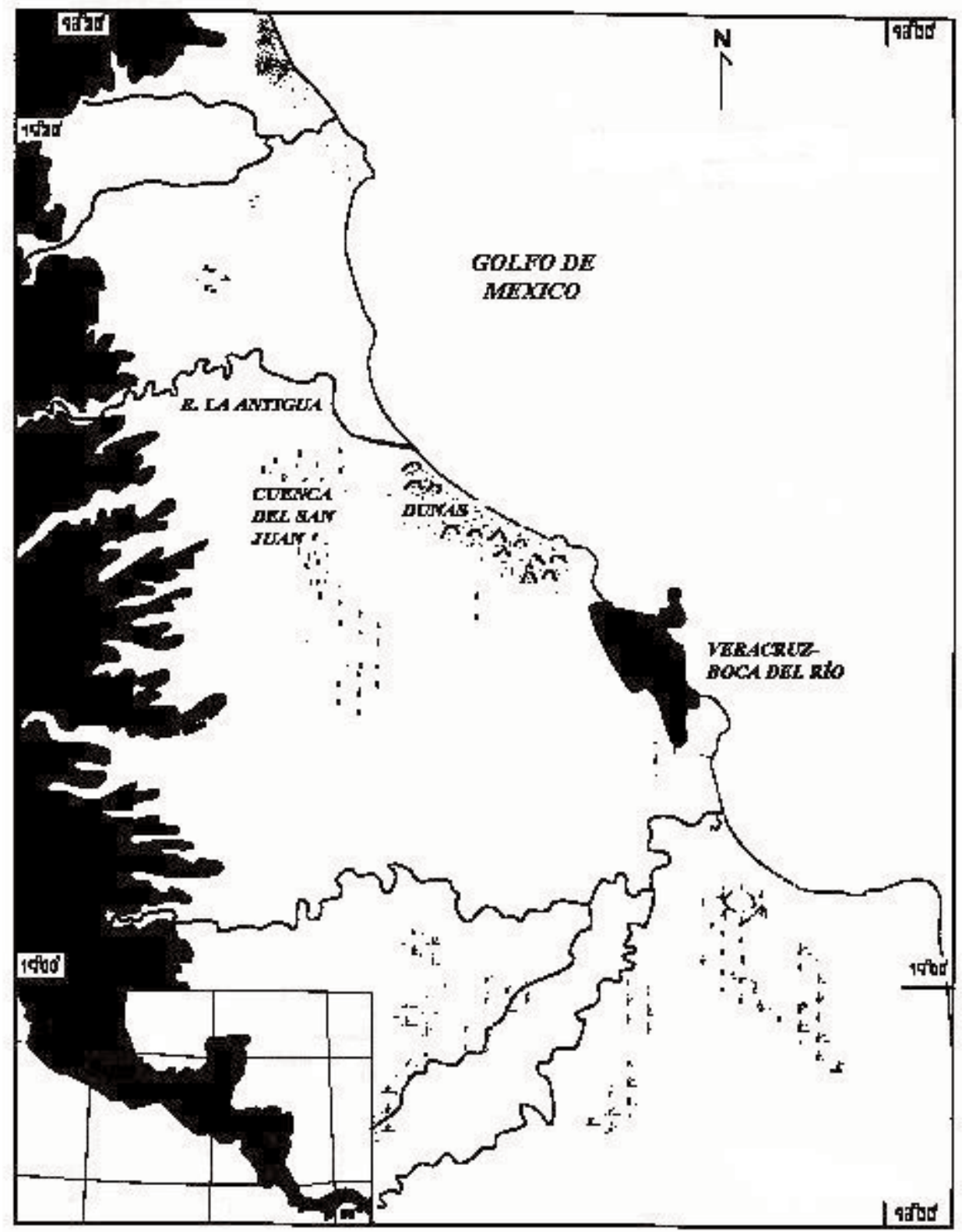

Figura 1. Localización de la región de estudio. Cuenca del Río San Juan y zona urbana Veracruz-Boca del Río, Veracruz, México. 2005. 
quienes establecieron en esta región las primeras explotaciones ganaderas e introdujeron el cultivo de la caña de azúcar (Hernández, 2001).

Las comunidades actuales de la cuenca detentan terrenos ejidales, y se cultiva caña de azúcar, maíz y ganadería bovina. También, existen grandes propiedades privadas con el giro ganadero.

El área urbana Veracruz-Boca del Río, es el núcleo poblacional más inmediato e importante, y constituye un polo de desarrollo que interactúa socioeconómicamente con las áreas rurales circundantes, ya que de cierta forma hay una afluencia de recursos de la ciudad al campo y viceversa. No solamente el área urbana es importante, sino también el circuito industrial representa un mercado de trabajo económicamente importante, pues parte de la población rural se emplea de manera fija o temporal en ella y se transporta diariamente a realizar trabajo asalariado.

Se aplicó un cuestionario estratificado al azar a 199 personas. Los estratos elegidos para filtrar los efectos de variables que no son motivo de este estudio, utilizan los criterios poblacionales de INEGI (2000): edad, residencia, sexo, educación e ingresos, similar a lo realizado por Turpie (2003) para valorar biodiversidad.

El cuestionario incluyó variables sociodemográficas, naturaleza (terrenos de ambientes silvestres), recursos naturales (bióticos, principalmente), ambiente (hábitat para las personas) y ganadería bovina (no estabulada), para explicar el valor del ambiente y de la ganadería, que se definen en la tabla 1 . Se pidieron opiniones sobre el ambiente regional y las políticas inherentes. Las preguntas de juicio se formularon como escalas de uno a diez, donde uno es la peor calificación y diez la mejor (Tabla 2). Se combinaron varias herramientas como el método de valoración contingente (MVC, Villa et al., 2002), con entrevistas con preguntas abiertas, trabajo de campo antropológico (Augé y Colleyn, 2005) e información secundaria. El objetivo de esta mezcla singular de métodos científicos y humanísticos fue capturar la complejidad de las percepciones ambientales y de su valoración en el contexto de un paisaje cultural regional (Saz et.al., 1998; Turpie, 2003; Clark et al., 2002; Pouta et al., 2002). Metodológicamente el estudio se fundamentó en la teoría de "bienestar total y libre albedrío", donde el argumento central es lograr que el público haga explícita su jerarquía de preferencias de bienestar (Hanemman, 1994; Hannon, 2001), expresando acuerdo o desacuerdo con opciones hipotéticas de gasto en programas de gobierno dirigidos a obtener un gradiente de posibles resultados en materia de uso pecuario del suelo, donde sólo uno de esos escenarios podría eventualmente darse y, en su caso, observarse los resultados esperados.

El argumento de este trabajo demanda que la elección libre de preferencia de una persona por algún programa o política de gobierno, conlleve su conformidad con las consecuencias de su elección, o sea, la aceptación de pros, contras e imponderables. Esta aproximación metodológica es una de las principales variantes de la valoración contingente, y como las otras, tiene como objetivo lograr una valoración de bienes y servicios públicos no cotizables en el mercado (Farber et al., 2002). Este valor se determina mediante cuestionarios con preguntas cuyas respuestas están predefinidas en clases cuantitativas que representan valores válidos según la teoría económica neoclásica, en pagos simulados por mejoras potenciales en el ambiente (Tisdell, 1993; Turpie, 2003). Cabe agregar que no se busca valorar la existencia de la cubierta vegetal que sostiene el pastoreo, sino sus cambios en atributos que inciden sobre el bienestar 
de los individuos (Saz et al., 1998). Este valor aparece, en el caso elegido en esta investigación, en unidades relativas que no son linealmente ligadas con valor económico, pero que son consistentes y establecen posiciones jerárquicas secuenciales de tipo marginal, equiparables al orden teórico del valor económico real de estos recursos (Chiesura y de Groot, 2003; Zhongwei et al., 2001).

Los datos de cada cuestionario fueron revisados en busca de errores e inconsistencias y fueron vaciados en una hoja de cálculo electrónica. El análisis de regresión logística se realizó mediante el procedimiento Logistic del programa estadístico SAS (Statistical Análisis System, SAS Inc), usando un modelo logit binario y el análisis de los estimadores de máxima verosimilitud. Por razones metodológicas, el orden ascendente habitual de los valores fue cambiado ( $0=$ Sí, $1=$ No, para las variables de respuesta).

El paso final fue el cuantificar DAP. Para ello hay que tomar en cuenta que las opiniones de valor se dieron sobre una escala arbitraria de 1 a 10, que para fines de análisis se equiparó a una escala de -4 a +5 , pues en las instrucciones a los entrevistados se les indicó que 5 era indiferencia. Una sumatoria de los valores marginales (de un individuo respecto al siguiente de menor valor pujado), nos daría un estimado del pago que la sociedad en su conjunto podría hacer en forma adicional a los flujos de efectivo que ya existen hoy en día, por obtener la mejoría del fenómeno estudiado. Este proceso se visualiza mejor modelando los valores de acuerdo a un modelo de superficie de tendencia (Agterberg, 1984) que manifieste con claridad la respuesta general de la disposición a pagar. La variable dependiente en este modelo fue el valor marginal otorgado (DAP) en escala arbitraria, y la independiente la población, obteniéndose para este caso un mejor ajuste con un polinomio de orden cuadrático. El área bajo la curva del modelo será la disposición a pagar por parte de la sociedad, estimada a partir de la muestra de personas entrevistadas.

\section{RESULTADOS}

\section{Aspecto social y demográfico}

Pese al mecanismo de estratificación, por efectos del azar la muestra contiene mayor cantidad de entrevistados del sexo masculino: $63,32 \%$ en la muestra, habiendo $47,15 \%$ hombres en la zona (INEGI, 2000), y también mayor frecuencia de edades de 21 a 40 años $(56,78 \%)$, con $13,07 \%$ de menor edad y el restante $30,15 \%$ de mayores de 40 años; ésta es una proporción razonable, dentro de la variabilidad que el azar introduce, pues según INEGI (2000), la región contiene $67,15 \%$ de adultos (>14 años). Aunque se procuró entrevistar a población representativa de todos los grados de escolaridad, ésta fue en promedio de grados de secundaria, no necesariamente terminada. Asimismo, predomina en la muestra la población urbana, que en esta región es de por sí ampliamente mayoritaria. La población urbana forma el $61,11 \%$ de la población de la zona y la rural el 38,69\%; $66,33 \%$ de la muestra provienen de Veracruz, 28,14\% de Boca del Río, 4,52\% son de La Antigua y $1,00 \%$ son visitantes provenientes de lugares lejanos. La proporción en la población regional, según INEGI (2000), es de $72,2 \%, 24,0 \%$ y $3,8 \%$, respectivamente.

El tiempo de residencia promedio fue de 9,7 años, con máximo de 60 años. Los ingresos promedio personales por mes fueron de $\$ 2,166.49$ y los ingresos familiares de $\$ 3,430$, aunque existen familias que obtienen desde un salario mínimo, hasta $\$ 30,000$ (Tabla 1). 


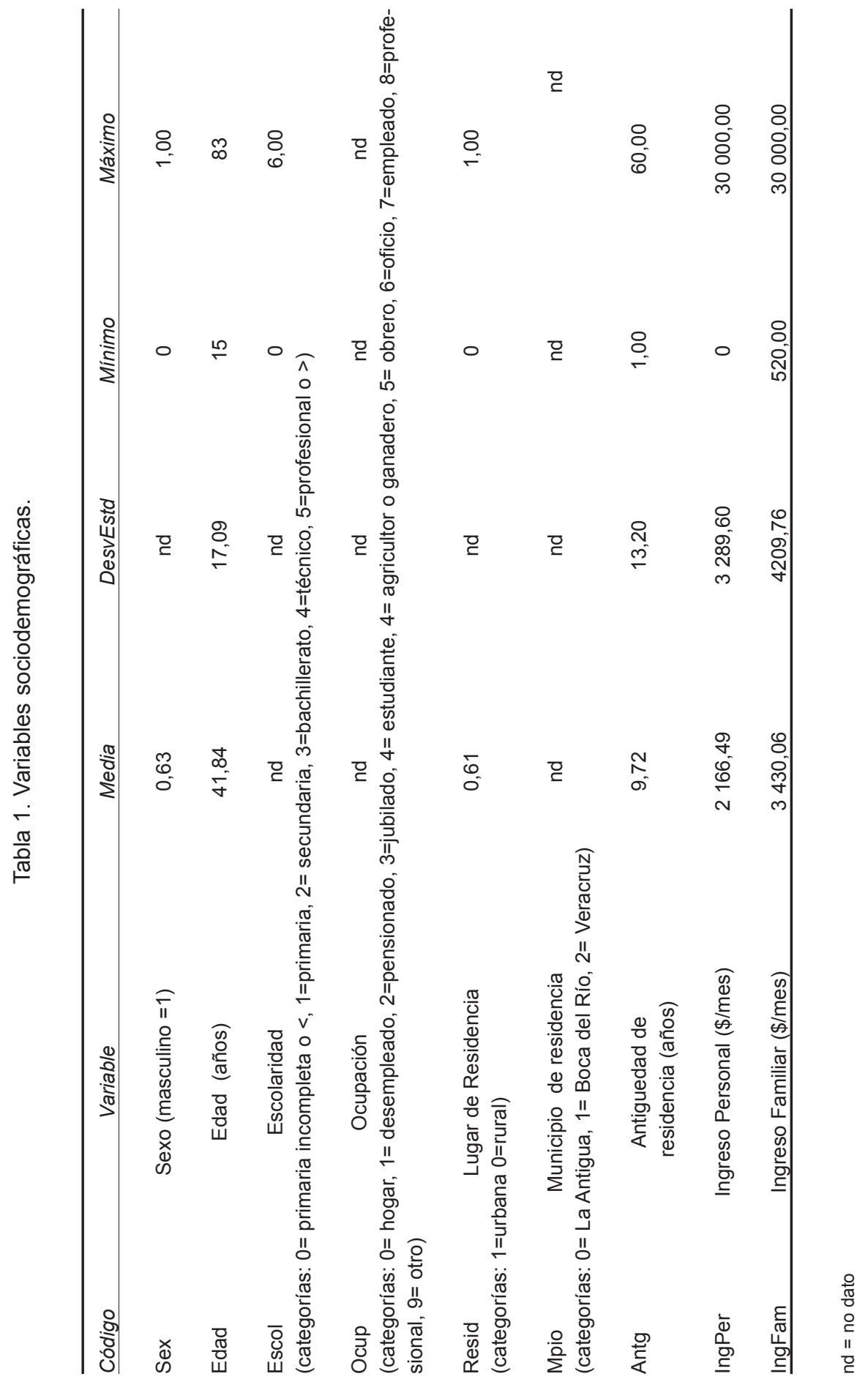




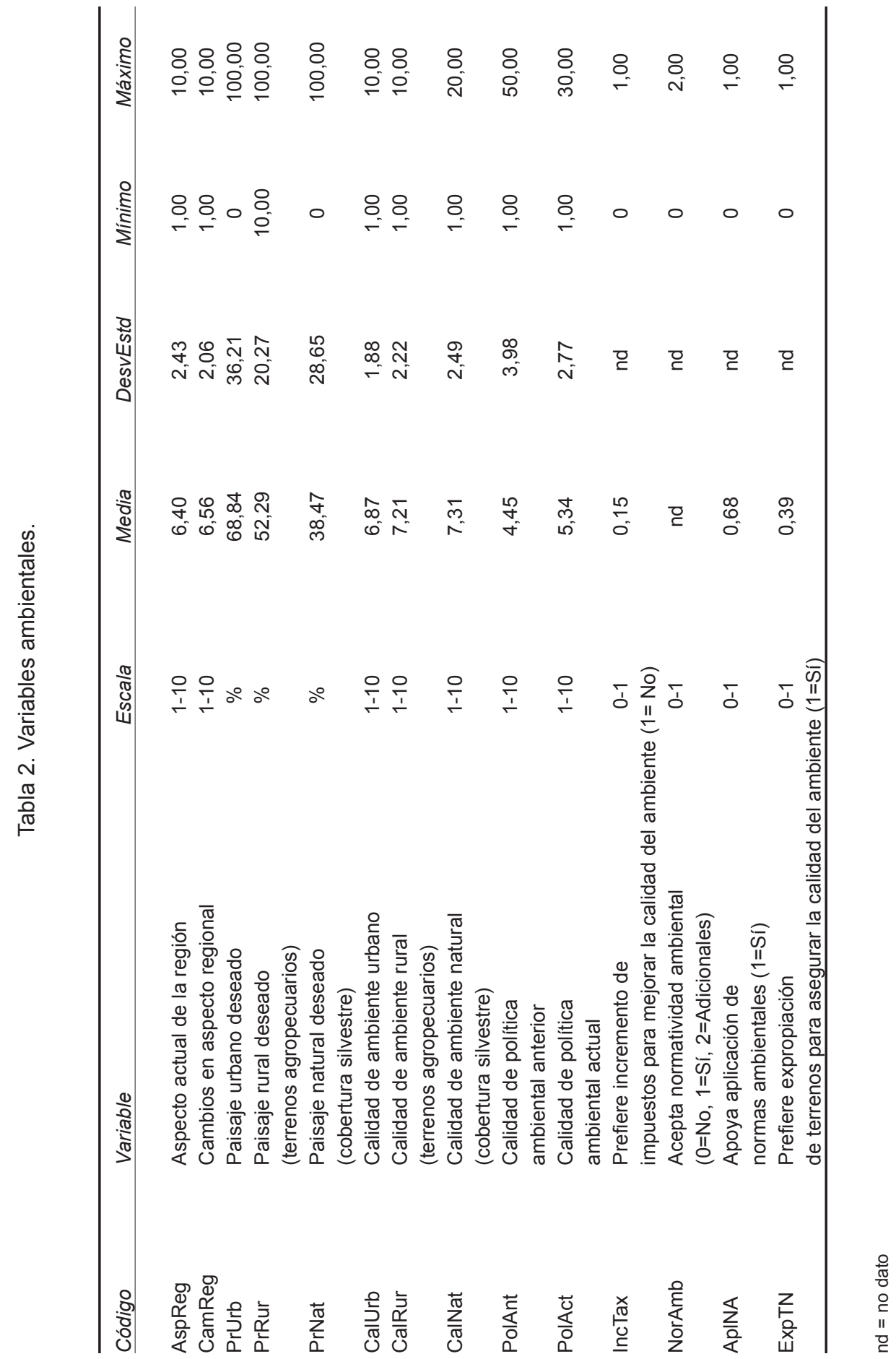




\section{Ambiente}

El aspecto de la región fue calificado como regular $(6,45 \pm 1,96)$, con tendencia a mejorar moderadamente $(7,41 \pm 3,46)$. Con respecto a la calidad ambiental del área urbana, ésta obtuvo una opinión de calidad buena $(7,48 \pm 2,48)$, mientras que el área rural y la naturalidad fueron calificadas con $6,74 \pm 2,41$ y 4,55 $\pm 2,30$, o sea, indiferente.

Con relación a política ambiental, los entrevistados a profundidad, con preguntas abiertas, señalaron que las desarrolladas por los gobiernos anteriores fueron malas, mientras que con las actuales son indiferentes, pues las opiniones vertidas externan que los gobiernos actuales (federal, estatal y municipal), aun no han sido capaces de ejecutar acciones que determinen un manejo adecuado e impactante en el ambiente.

Posteriormente, cuando se les preguntó si apoyarían que el gobierno incrementara recaudación fiscal a favor del mejoramiento de la calidad ambiental, la mayoría aceptó $(84,42 \%)$. De la misma forma, la población observa una actitud positiva con relación al apoyo para la promulgación $(82,91 \%$ piden más legislación, 13,07\% aceptan la que hay, 4,02\% piden menos), y procuración de normas que mejoren la calidad ambiental $(67,84 \%$ piden aplicación rigurosa). Finalmente, no se observó gran apoyo hacia la expropiación para conservar las áreas de la zona $(38,90 \%)$.

\section{Recursos naturales}

Los recursos naturales de la región fueron calificados como buenos y abundantes $(7,58 \pm 2,14)$, y la mayor parte de los entrevistados manifestaron un tibio apoyo a la existencia de normas para mejorarlos $(63,32 \%)$, y escaso apoyo a una posible expropiación de los terrenos para asegurar calidad de los recursos naturales $(39,90 \%)$.

\section{Ganadería}

Los entrevistados calificaron el aspecto y calidad de los agostaderos de la región como buenos $(6,45 \pm 1,96)$. Sin embargo, la mayoría desconoce la existencia de las normas ambientales específicas para las actividades ganaderas $(94,97 \%)$, si bien la mayor parte consideró que se debe actuar en función de una cierta normatividad ambiental $(83,42 \%)$. Además, una minoría en la muestra no está de acuerdo en que la normatividad sea tan rigurosa $(35,18 \%$ están en contra del rigor; $63,82 \%$ piden enérgica procuración, y $1 \%$ indiferente). Pocos consideraron que reviste importancia el expropiar si los terrenos fuesen vulnerables $(39,90 \%)$. Por otro lado, gran parte de la muestra $(90,45 \%)$ consideró que es conveniente subsidiar a los ganaderos predispuestos a cumplir voluntariamente con la normatividad (Tabla 4). Al respecto, Chiesura y de Groot (2003) señalan que no podemos esperar que el bienestar sea determinado como una respuesta con racionalidad económica. Sin embargo, estudios realizados en Suecia por Lewan y Soderquist (2002), señalaron que los entrevistados reconocen más los servicios visibles de los ecosistemas. Esto se vincula al hecho de que la valoración de la naturaleza y los servicios que presta, al no tener mercado, los hace invisibles a los sistemas económicos que la población siempre tiene presente. Lo anterior indica que las preferencias humanas están vinculadas con la calidad del conocimiento e información a través de la propaganda de la que se sirven tanto las instituciones formales como las informales. 


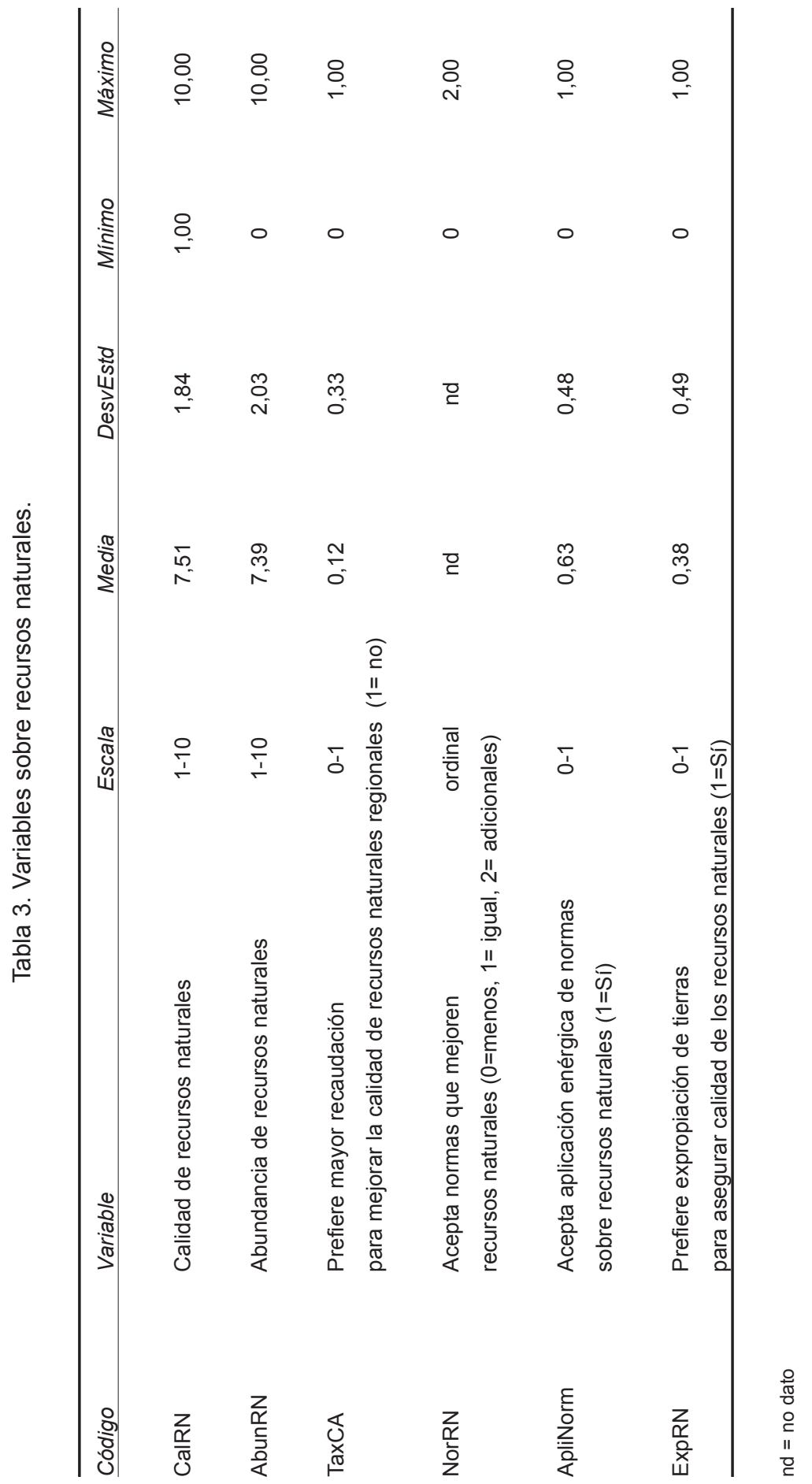




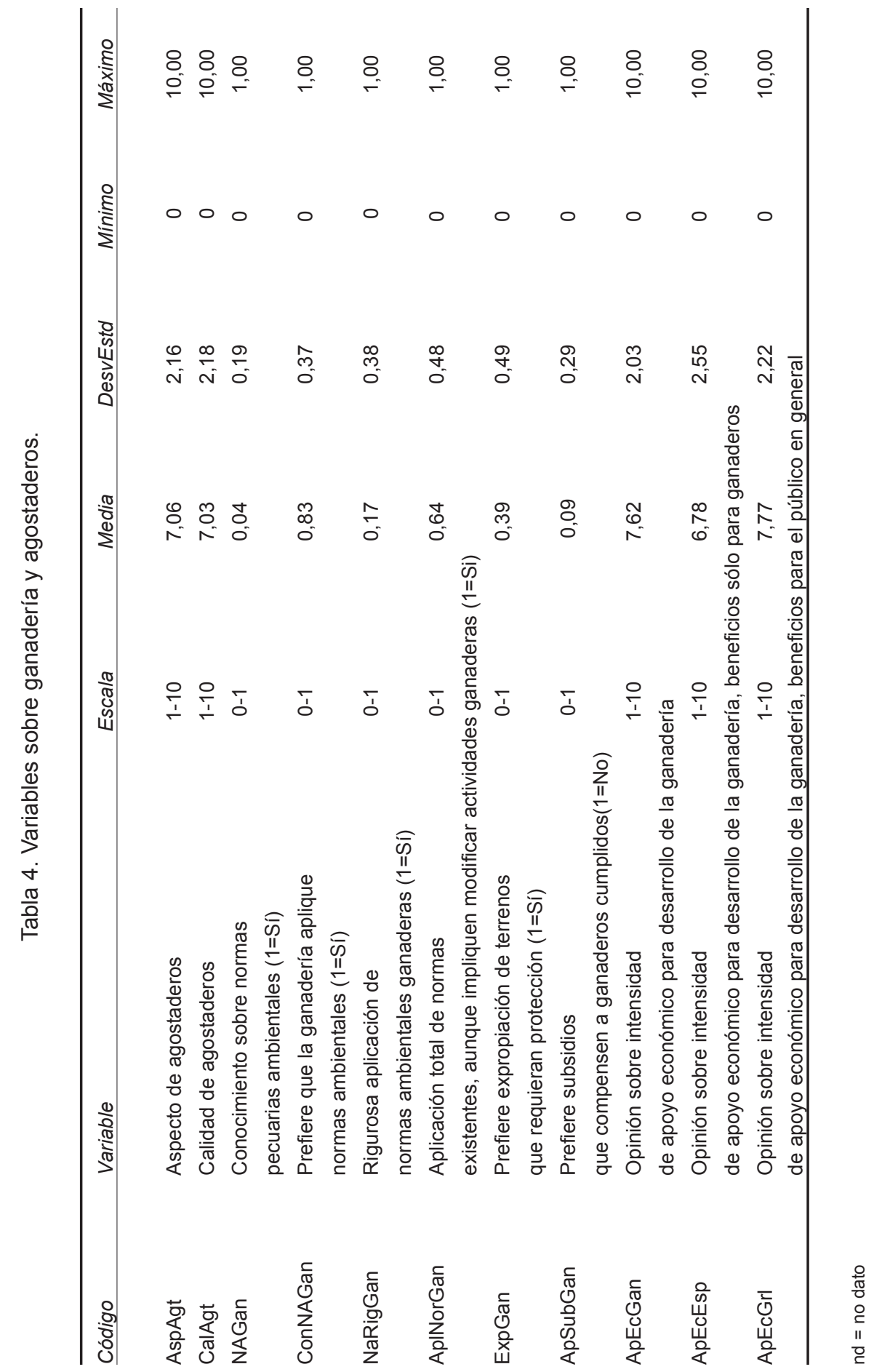


Tabla 5. Regresión logística de incremento impositivo para programas de mejoramiento del ambiente (IncTax) vs. variables estudiadas.

\begin{tabular}{|c|c|c|c|}
\hline VARIABLE & ESTIMADOR & WALD $(G L=1)$ & PROBABILIDAD \\
\hline \multicolumn{4}{|c|}{ Variables sociodemográficas } \\
\hline Sex & 0,000372 & 0,6986 & 0,9996 \\
\hline Edad & 0,0633 & 0,2607 & 0,8082 \\
\hline Escol & 0,0723 & 0,1945 & 0,7102 \\
\hline Ocup & 0,1382 & 0,2085 & 0,5075 \\
\hline Resid & $-0,8383$ & 0,5625 & 0,1362 \\
\hline Mpio & 0,5047 & 0,2897 & 0,0814 \\
\hline Antg & $-0,00394$ & 0,0188 & 0,8344 \\
\hline IngPer & $-0,00092$ & 0,000409 & $0,0245^{*}$ \\
\hline IngFam & 0,00109 & 0,000437 & $0,0122^{*}$ \\
\hline \multicolumn{4}{|c|}{ Variables ambientales } \\
\hline AspReg & $-0,2695$ & 6,0907 & 0,9647 \\
\hline CamReg & 1,6527 & 5,4306 & 0,7609 \\
\hline PrUrb & 0,4624 & 1,8933 & 0,8070 \\
\hline PrRur & $-0,5041$ & 0,6392 & 0,4303 \\
\hline PrNat & 0,2686 & 0,7379 & 0,7158 \\
\hline CalUrb & 0,7785 & 7,9979 & 0,9225 \\
\hline CalRur & $-0,8512$ & 14,4035 & 0,9529 \\
\hline CalNat & 3,7990 & 10,2792 & 0,7117 \\
\hline PolAnt & $-0,6775$ & 4,7676 & 0,8870 \\
\hline PolAct & $-4,1313$ & 4,0468 & 0,3073 \\
\hline NorAmb & 5,8044 & 17,6913 & 0,7428 \\
\hline ApINA & $-1,4475$ & 21,5730 & 0,9465 \\
\hline ExpTN & 28,4044 & 52,9505 & 0,5917 \\
\hline \multicolumn{4}{|c|}{ Variables sobre recursos naturales } \\
\hline CalRN & 0,2040 & 0,1609 & 0,2048 \\
\hline AbunRN & 0,0541 & 0,1587 & 0,7331 \\
\hline NorRN & $-2,1701$ & 0,4446 & $<.0001^{* *}$ \\
\hline ApliNorm & 0,5587 & 0,6331 & 0,3775 \\
\hline ExpRN & 0,5714 & 0,6979 & 0,4129 \\
\hline \multicolumn{4}{|c|}{ Variables sobre ganadería y agostaderos } \\
\hline AspAgt & $-0,2246$ & 0,2142 & 0,2943 \\
\hline CalAgt & 0,2239 & 0,2146 & 0,2968 \\
\hline NAGan & 0,8338 & 1,2126 & 0,4917 \\
\hline ConNAGan & $-0,3190$ & 0,9138 & 0,7270 \\
\hline NaRigGan & 2,8805 & 1,1342 & $0,0111^{*}$ \\
\hline ApINorGan & 1,5352 & 0,5881 & $0,0090^{* *}$ \\
\hline ExpGan & 0,4980 & 0,6466 & 0,4412 \\
\hline ApEcGan & 0,2579 & 0,1638 & 0,1153 \\
\hline ApEcEsp & $-0,0632$ & 0,1366 & 0,6436 \\
\hline ApEcGrl & $-0,1182$ & 0,1275 & 0,3540 \\
\hline
\end{tabular}

* Significativa con $p<0,05$

** Significativa con $p<0,01$ 
Regresión logística para una política de mayor recaudación (IncTax)

Las variables socioeconómicas ingresos personales (IngPer) y familiares (IngFam) fueron determinantes en términos de la disponibilidad de apoyo al incremento de impuestos (IncTax), si estos recursos fuesen etiquetados exprofeso para mejorar la calidad del ambiente $(p<0,05)$. Otras variables altamente significativas $(p<0,05)$ fueron: apoyo a la existencia de normas que mejoren los recursos naturales regionales (NorRN), así como la necesidad de tener suficiencia y rigor en las normas ambientales para ganadería (NaRigGan) y la aplicación total de las normas existentes (ApIRigGan), aunque impliquen modificar o reducir las actividades en terrenos ganaderos (modificación de sistemas productivos como tipo de tecnología usada). Según la Tabla 5 , Spash (2000) señaló la influencia del nivel de ingreso, y Mansky (2000) sugiere que en este tipo de valoraciones resaltan aspectos subjetivos como expectativas, que surgen por diferencias en ingresos, niveles de consumo y educación.

\section{Regresión logística para reasignación presupuestal (TaxCa)}

La variable "Apoyo a mayor uso de impuestos para mejorar la calidad de recursos naturales regionales" (TaxCa), implica mayor disponibilidad presupuestal para los programas ambientales del gobierno, sin incrementar el total de contribuciones de ningún ciudadano. La variable socioeconómica de más impacto sobre esta variable de respuesta fue el municipio de residencia (Mpio. La Antigua con menor disposición, Veracruz y Boca del Río no significativas). Otras variables estadísticamente significativas $(p<0,05)$ fueron la necesidad de normas que mejoren los recursos naturales regionales (NorRN). En materia de ambientes silvestres las variables relevantes fueron el apoyo a la aplicación total de las normas existentes (ApliNorm), aunque impliquen modificar o reducir las actividades en terrenos ganaderos. El soporte a la intensidad de apoyo económico para desarrollo de la ganadería ambientalmente responsable (ApEcGrl), fue estadísticamente importante dentro de las variables relacionadas a ganadería y pastizales (Tabla 6).

\section{Regresión logística para subsidios a la ganadería ambientalmente respon- sable (ApSubGan)}

Las variables que tuvieron significancia estadística $(p<0,05)$ fueron: residencia (rural o urbana, Resid), y apoyo a la aplicación de normas ambientales (ApINA) (Tabla 7).

\section{Disposición a pagar}

En la Figura 2 se presentan las ecuaciones ajustadas de los valores marginales sobre la disposición al pago de subsidios en ganadería y ambiente, en relación con el sentir de la población. Para interpretar las gráficas de la Figura 2 hay que considerar que en el caso de la ganadería ambientalmente responsable, 171 de 199 entrevistados $(85,93 \%)$ están dispuestos a pagar, mediante diversos mecanismos de pago, cantidades positivas por los costos sombra adicionales que implica esta opción de manejo ganadero, y sólo 28 consideran una disposición de pago negativa (que desearían les fuera devuelta por los mismos mecanismos de pago). En tanto $166(83,42 \%)$, están dispuestos a pagar cantidades positivas por asegurar un mejor ambiente. En el caso ganadero 
Tabla 6. Regresión logística de incremento impositivo para mejoramiento de la calidad de los recursos naturales (TaxCA) vs. variables estudiadas.

\begin{tabular}{|c|c|c|c|}
\hline VARIABLE & ESTIMADOR & WALD (GL=1) & PROBABILIDAD \\
\hline \multicolumn{4}{|c|}{ Variables sociodemográficas } \\
\hline Sex & $-0,3102$ & 0,8957 & 0,7291 \\
\hline Edad & $-0,1967$ & 0,2821 & 0,4857 \\
\hline Escol & 0,3622 & 0,2670 & 0,1749 \\
\hline Ocup & 0,00144 & 0,2983 & 0,9961 \\
\hline Resid & 0,7990 & 0,6547 & 0,2223 \\
\hline Mpio & 0,7677 & 0,3271 & $0,0189^{*}$ \\
\hline Antg & $-0,00420$ & 0,0220 & 0,8488 \\
\hline IngPer & 0,000170 & 0,000340 & 0,6163 \\
\hline IngFam & 0,000069 & 0,000273 & 0,8007 \\
\hline \multicolumn{4}{|c|}{ Variables ambientales } \\
\hline AspReg & 0,0823 & 0,3550 & 0,8166 \\
\hline CamReg & $-0,5510$ & 0,4398 & 0,2102 \\
\hline PrUrb & $-0,1115$ & 0,0761 & 0,1428 \\
\hline PrRur & $-0,1031$ & 0,0779 & 0,1856 \\
\hline PrNat & $-0,0682$ & 0,0636 & 0,2835 \\
\hline CalUrb & 0,3728 & 0,3268 & 0,2539 \\
\hline CalRur & 0,2177 & 0,5830 & 0,7089 \\
\hline CalNat & $-0,3257$ & 0,4618 & 0,4805 \\
\hline PolAnt & 0,0503 & 0,1914 & 0,7926 \\
\hline PolAct & 0,0837 & 0,3183 & 0,7927 \\
\hline NorAmb & 6,6092 & 4,0712 & 0,1045 \\
\hline ApINA & 0,7537 & 1,1837 & 0,5243 \\
\hline ExpTN & 0,2520 & 1,4877 & 0,8655 \\
\hline \multicolumn{4}{|c|}{ Variables sobre recursos naturales } \\
\hline CalRN & $-0,00521$ & 0,2057 & 0,9798 \\
\hline AbunRN & 0,3937 & 0,2196 & 0,0730 \\
\hline NorRN & $-3,6270$ & 0,6976 & $<, 0001^{* *}$ \\
\hline ApliNorm & 0,7661 & 0,8769 & 0,3823 \\
\hline ExpRN & 1,6431 & 1,1153 & 0,1407 \\
\hline \multicolumn{4}{|c|}{ Variables sobre ganadería y agostaderos } \\
\hline AspAgt & $-0,2737$ & 0,2410 & 0,2560 \\
\hline CalAgt & 0,3053 & 0,2354 & 0,1947 \\
\hline NAGan & $-1,8049$ & 1,0605 & 0,0888 \\
\hline ConNAGan & $-2,2671$ & 1,2492 & 0,0695 \\
\hline NaRigGan & 0,3474 & 0,7331 & 0,6356 \\
\hline ApINorGan & 2,1589 & 0,7335 & $0,0032^{* *}$ \\
\hline ExpGan & $-0,0280$ & 0,8881 & 0,9749 \\
\hline ApEcGan & 0,0968 & 0,1624 & 0,5510 \\
\hline ApEcEsp & $-0,0293$ & 0,1494 & 0,8444 \\
\hline ApEcGrl & 0,490 & 0,1309 & $0,0076^{* *}$ \\
\hline
\end{tabular}

* Significativa con $p<0,05$

** Significativa con $p<0,01$ 
Tabla 7. Regresión logística para subsidio a la ganadería ambientalmente responsable (ApSubGan) vs. variables estudiadas.

\begin{tabular}{|c|c|c|c|}
\hline VARIABLE & ESTIMADOR & WALD $(G L=1)$ & PROBABILIDAD \\
\hline \multicolumn{4}{|c|}{ Variables sociodemográficas } \\
\hline Sex & 0,9053 & 1,0945 & 0,4082 \\
\hline Edad & $-0,4310$ & 0,3533 & 0,2225 \\
\hline Escol & $-0,1212$ & 0,2899 & 0,6759 \\
\hline Ocup & 0,1012 & 0,3423 & 0,7674 \\
\hline Resid & 2,0507 & 0,9487 & $0,0307^{*}$ \\
\hline Mpio & 0,6593 & 0,3679 & 0,0731 \\
\hline Antg & 0,00951 & 0,0254 & 0,7081 \\
\hline IngPer & $-0,00179$ & 0,00141 & 0,2032 \\
\hline IngFam & 0,00184 & 0,00143 & 0,1982 \\
\hline \multicolumn{4}{|c|}{ Variables ambientales } \\
\hline AspReg & 0,6800 & 0,5101 & 0,1825 \\
\hline CamReg & 0,4650 & 0,2548 & 0,0680 \\
\hline PrUrb & 0,0475 & 0,0852 & 0,5768 \\
\hline PrRur & 0,0862 & 0,1092 & 0,4299 \\
\hline PrNat & 0,0322 & 0,0907 & 0,7223 \\
\hline CalUrb & $-0,2556$ & 0,2540 & 0,3143 \\
\hline CalRur & 0,6173 & 0,6631 & 0,3519 \\
\hline CalNat & $-0,6960$ & 0,6541 & 0,2873 \\
\hline PolAnt & $-0,5611$ & 0,3398 & 0,0987 \\
\hline PolAct &, 2174 & 0,6702 & 0,0693 \\
\hline NorAmb & $-8,0318$ & 5,9516 & 0,1772 \\
\hline ApINA & 3,9896 & 1,7972 & $0,0264^{*}$ \\
\hline ExpTN & $-2,1346$ & 1,9733 & 0,2794 \\
\hline \multicolumn{4}{|c|}{ Variables sobre recursos naturales } \\
\hline CaIRN & 0,2342 & 0,1634 & 0,1518 \\
\hline AbunRN & 0,00519 & 0,1584 & 0,9739 \\
\hline NorRN & 0,0211 & 0,4670 & 0,9639 \\
\hline ApliNorm & 0,5034 & 0,6339 & 0,4271 \\
\hline ExpRN & 0,7072 & 0,7317 & 0,3338 \\
\hline \multicolumn{4}{|c|}{ Variables sobre ganadería y agostaderos } \\
\hline AspAgt & $-0,447$ & 0,3175 & 0,6485 \\
\hline CalAgt & $-0,323$ & 0,2975 & 0,6566 \\
\hline NAGan & $-0,555$ & 1,3972 & 0,9114 \\
\hline ConNAGan & 1,6710 & 1,0279 & 0,1040 \\
\hline NaRigGan & $-0,7017$ & 0,6869 & 0,3069 \\
\hline ApINorGan & 0,2415 & 0,7254 & 0,7392 \\
\hline ExpGan & 0,3861 & 0,9751 & 0,6921 \\
\hline ApEcGan & 0,3924 & 0,2016 & 0,0516 \\
\hline ApEcEsp & 0,3440 & 0,1708 & $0,0439 *$ \\
\hline ApEcGrl & $-0,2369$ & 0,1608 & 0,1407 \\
\hline
\end{tabular}

* Significativa con $p<0,05$

** Significativa con $p<0,01$ 
se estima un pago neto, sumando algebraicamente pagos positivos $(58,840)$ y negativos (292), de 58,548 unidades de valor, lo cual es equivalente a una proporción de beneficios respecto a costos sociales de 201 a 1. Para el caso de ambiente serían pagos positivos de 72,277 menos 715 pagos negativos, dando un valor neto de 61,562 unidades de valor, o sea, una relación de 101 a 1 en beneficios respecto a los costos a la sociedad.

De estos valores, suavizados por el modelo de superficie de tendencia, se entiende que la mayor parte de la población está de acuerdo en que los ganaderos reciban algún tipo de subsidio por practicar su actividad sin deteriorar el ambiente, como se observa en el área positiva de la Figura 2B. Situación similar ocurre con el valor que le da la población a la conservación del ambiente (Figura 2A), en donde la mayoría de la población está consciente de los altos niveles de deterioro ambiental de la zona y de la necesidad de introducirlo dentro de sus satisfactores para lograr una buena calidad de vida.

\section{DISCUSIÓN}

Un aspecto importante en la aplicación de la cédula de la entrevista fue introducir a los entrevistados en un total entendimiento del significado de ambiente y las escalas de valor consideradas, para que las respuestas generaran un acercamiento más preciso de las preferencias y el nivel de satisfacción que obtienen del ambiente actual, del uso del suelo y de la vegetación. Esta situación fue reforzada por entrevistas a profundidad, donde se permitió al entrevistado extenderse. Se partió del hecho que el valor es un término relativo, que refleja el contexto en el cual la valoración tuvo lugar, y la percepción que la población asignó a los valores establecidos. Cada asignación es una indicación del valor y la importancia que el bien analizado, en este caso la ganadería, tiene para los entrevistados (Brown, 1984).

Pareciera que la mayoría de los entrevistados opinan en favor de las políticas que no comprometen lo individual, o donde ellos no se vean impactados en forma directa, como es el caso de los subsidios al productor, los cuales se supone deben correr a cargo del gobierno. Al respecto, Chiesura y de Groot (2003) señalan que no podemos esperar que los niveles de bienestar que los entrevistados perciben del bien en cuestión sean determinados como una respuesta con racionalidad económica, ya que la gente tiende a buscar seguridad personal o individual. Los entrevistados argumentaron que las instancias de gobierno son las que deben ejecutar las acciones para conservar la naturaleza y que la sociedad no debe desembolsar dinero de forma directa, ya que los recursos públicos provienen del pueblo. Sadoulet y de Janvry (1999) señalan que las acciones de las personas y las empresas pueden responder en forma amplia a políticas gubernamentales de liderazgo y de riesgo compartido en intentos para rediseñar productos, procesos de producción y consumo, para evitar o para reducir los efectos negativos que se desean controlar. Desde luego, las acciones de liderazgo moral y los subsidios no son excluyentes, toda vez que en diversos lugares del mundo se han constituido canastas de instrumentos de política ambiental que actúan concurrentemente, representando también una posibilidad para el futuro cercano de México.

Por otra parte, el estudio mostró que la DAP local para protección del ambiente y el paso a una ganadería responsable se incrementa o reduce de acuerdo con los niveles de ingreso que los entrevistados obtienen. Una situación similar fue encontrada por Turpie (2003) en Sudáfrica, en 


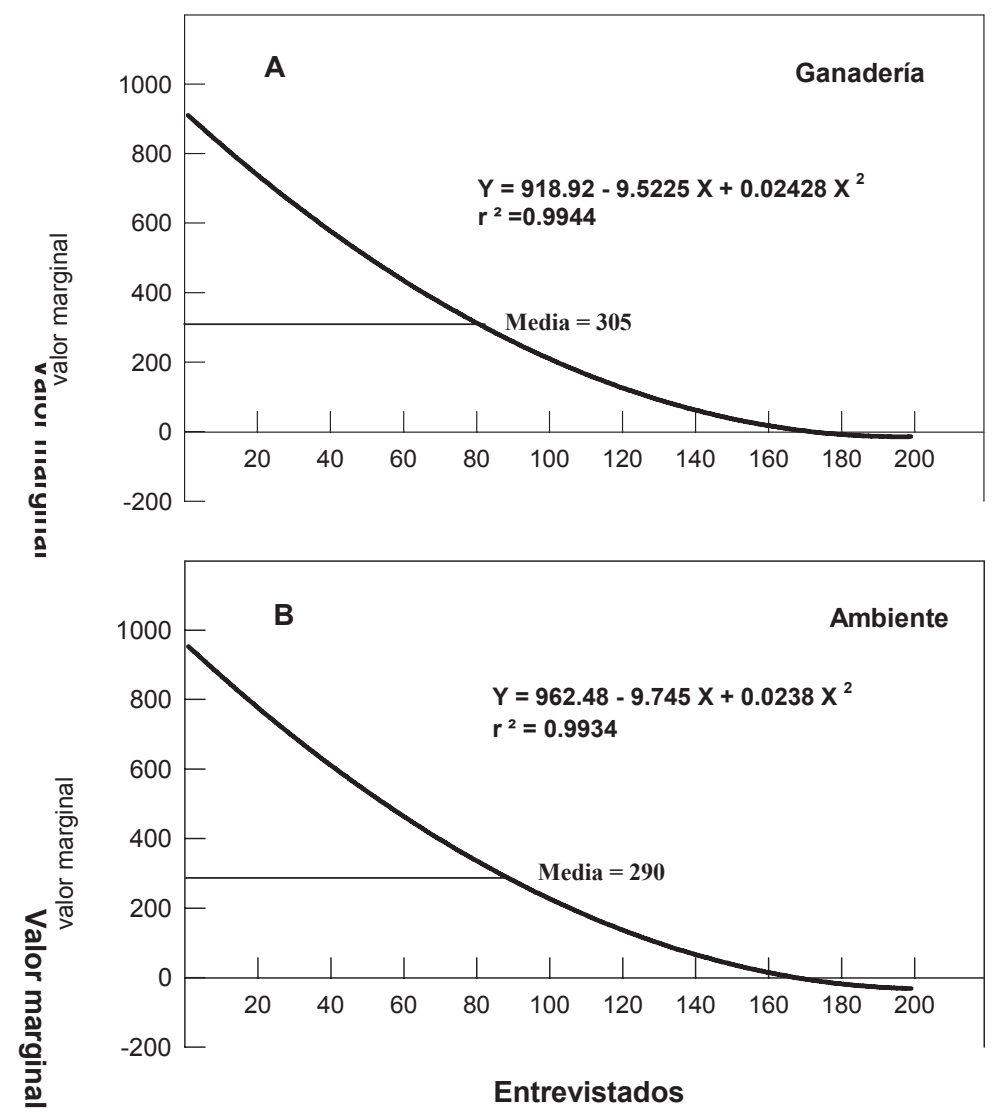

Figura 2. Valores marginales otorgados por la población sobre la disposición al pago por: A) actividades ganaderas conservacionistas y B) conservación del ambiente.

relación con la protección de la biodiversidad. A su vez, Spash (2000) señaló la importancia de los procesos sociales en la valoración ambiental, ya que la disposición a pagar se ve fuertemente influida por los niveles de ingreso, y las personas concederán valor o precio a productos 0 servicios de acuerdo a lo que ellos mismos consideren que no disminuirá su nivel de vida actual. De la misma forma, las entrevistas mostraron que las personas de menores ingresos observan DAP menor en virtud de que sus prioridades se orientan a satisfacer sus necesidades de sobrevivencia. La disposición negativa a pagar por un bien o servicio puede originarse en niveles de ingreso bajos. En este sentido, si las preferencias no son intercambiables por su actual nivel de vida aparece la selección de un escenario. Lo anterior puede clasificarse como un proceso de decisión no compensatoria, lo cual implica que existen bienes cuyo nivel de bienestar no es intercambiable, dentro de los cuales aparece en forma inmediata el nivel de vida. Mainwaring (2001) consideró que también las preferencias sociales ejercen un fuerte peso en la disposición a pagar, pues son de índole moral, y deben ser analizadas 
como externalidades. En este caso, señala que las preferencias entre los individuos con diferentes posiciones difieren no sólo por los niveles de ingreso, sino también por los de consumo. Una persona con bajo nivel de ingreso puede señalar una disposición a pagar alta, pero no es comparable con la de una persona con ingresos altos, ya que sus niveles de consumo son indistintos y su percepción de valor también.

\section{CONCLUSIONES}

En términos generales, se encontró una actitud favorable entre los entrevistados hacia el incremento de impuestos, apoyos y subsidios gubernamentales que permitan mejorar la calidad del ambiente y los recursos naturales, y que compensen adecuadamente a los ganaderos que cumplan con las metas de calidad ambiental prescritas. Se detectó que predomina un apoyo hacia la estricta aplicación de la legislación vigente sobre el particular, así como a la potencial creación de normas complementarias que mejoren los recursos naturales y el ambiente regional. No obstante, se percibe que el conocimiento general que la población posee de las normas que aplican para el ambiente, los recursos naturales y la ganadería, es deficiente e incompleto, en términos de sus disposiciones generales, ámbito de competencia, alcances y limitaciones.

Se encontró una disposición de pago positiva por calidad ambiental y recursos naturales de la región, sin embargo, ésta debe ser vía impuestos que el gobierno debe destinar ex profeso.

Se pudo observar que el público mantiene su interés en la ganadería, llegando a proponer apoyos adicionales para su desarrollo. La ganadería es una actividad importante que por siglos ha ayudado a construir el actual paisaje regional, por lo que no sorprende que el público local lo considere un paisaje deseable, sin que ello le exima de trabajar bajo normatividad que permita reducir el deterioro de la calidad ambiental. Se considera apropiado premiar a los ganaderos que cumplan con las metas de calidad ambiental por medio de subsidios. Por lo mismo, la percepción del nivel de bienestar de la población con relación a la calidad ambiental y los servicios prestados por las praderas es buena.

\section{REFERENCIAS}

Agterberg, F.P. 1984. Trend surface analysis. In: G.L. Gaile and C.J. Willmott, eds. Spatial statistics and models. Reidel, Dordrecht, Holanda. p. 147-171.

Augé, M. y J.P. Colleyn. 2005. Qué es la antropología. Paidós, Barcelona, España. 138 p.

Brown, T. C. 1984. The concept of value in resource allocation. Land Economics 60 (3): 231-246.

Capalbo, S.M. y J.M Antle. 1989. Incorporating social costs in the returns of agricultural researches. American Journal of Agricultural Economics 71 (2): 458463.

Clark J., J. Burgess y C.M. Harrison. 2002. I struggled with this money business: respondents' perspectives on contingent valuation. Ecological Economics 33: 45-62.

Chiesura A. y R. de Groot. 2003. Critical natural capital: a socio-cultural perspective. Ecological Economics 44: 219231.

Del Ángel P., A.L. 1994. Formación de la estructura productiva ganadera en la llanura costera de Veracruz central. In: 
E. Velázquez y O. Hoffman. Llanuras Costeras de Veracruz, La Lenta Construcción de Regiones, ORSTOM, CIESAS, Universidad Veracruzana.

INEGI. 2000. Fuente de datos de población: INEGI-XII Censo de Población y Vivienda 2000, completo; http;//www.inegi.gob.mx

Farber, M.A., R. Constanza y M.A. Wilson. 2002. Economic and ecological concepts for valuing ecosystem services. Ecological Economics 41:375-392.

García, E. 1988. Modificaciones al sistema de clasificación climática de Köppen. E. García (Ed.) México.

Hanemman, W.M. 1994. Valuing the environment through contingent valuation. Economic Perspectives 8 (4):19 - 43.

Hannon, B. 2001. Ecological pricing and economic efficiency. Ecological Economics 36: 19-30.

Hernández, L. (comp.). 2001. Historia ambiental de la ganadería en México. L'Institut de Recherche pour le Développement, Instituto de Ecología, Xalapa, Veracruz. 276 p.

Kondratyev, K.Y., F. Moreno P. e I. Galindo. 1997. Desarrollo sustentable y dinámica de población. Universidad de Colima, Colima. 136 p.

Lewan, L. y T. Soderquist. 2002. Knowledge and recognition of ecosystem services among the general public in a drainage basin in Scania, Southern Sweden. Ecological Economics 42: 459-467.

Macmillan, D.C., L. Philip, N. Hanley y B. Álvarez. 2002. Valuing the non market benefits of wild goose conservation: a comparison of interview and group- based approaches. Ecological Economics 43:49-59.

Mainwaring, L. 2001. Environmental values and the frame of reference. Ecological Economics 38: 391-402.

Mansky, C. F. 2000. Economics analysis of social interactions. The Journal of Economics Perspectives 14 (3):115136.

Nasi, R., S. Wunder y J.A. Campos. 2002. Forest ecosystem services: can they pay our way out of deforestation? A discussion paper prepared for the GEF, Forestry Roundtable, in conjunction with the UNFF II, Costa Rica, March 11, 2002.

Pouta, E., M. Rekola, J. Kuuluvainen, L. Chuan-Zhong y O. Tahvonen. 2002. Willingness to pay in different policyplanning methods: insights into respondents' decision-making processes. Ecological Economics 40:295-311.

Romo Lozano, J.L. 2005. Valuación económica de la migración de las mariposas monarca. Instituto Nacional de Ecolog'a, México. http://www.ine.gob. mx/ueajei/publicaciones/libros/286/rom o.html. Consultado 18 jun 2005.

Sadoulet, E. y A. de Janvry. 1999. Quantitative development policy analysis. The Johns Hopkins University Press, Baltimore. 397 p.

SAGAR. 1999. Programa Nacional Pecuario (completo). Secretaría de Agricultura Ganadería y Desarrollo Rural. México, D.F. 16 p.

Sarabia Bueno, C.C. 2004. Sistema lagunar de la Ciudad de Veracruz, México. Propuesta de manejo bajo la visión de proyectación y gestión ambiental. Disertación doctoral, Colegio de 
Posgraduados, Campus Veracruz, Veracruz. 306 p.

Saz Z., S., L. Pérez P. y J. Barreiro H. 1998. Valoración contingente y protección de espacios naturales. RVEA 23, Madrid. $144 \mathrm{p}$.

Siemens, A.H. 1998. A favored place. San Juan River wetlands, Central Veracruz, A.D. 500 to the present. University of Texas Press. 301 p.

Soderqvist, T. 2003. Are farmers prosocial? Determinants of the willingness to participate in a Swedish catchment-based wetland creation programme. Ecological Economics 47:105-120

Spash, C. L. 2000. Ecosystems, contingent valuation and ethics: the case of wetland re-creation. Ecological Economics 34:195-215

Stevens, T.H., R. Belkner, D. Dennis, D. Kittredge y C. Willis. 2000. Comparison of contingent valuation and conjoint analysis in ecosystem management. Ecological Economics 32:63-74
Tisdell, C.A. 1993. Economics of environmental conservation. Economics for enviromental \& ecological management. Elsevier Science Publications. Amsterdam. $233 \mathrm{p}$.

Turpie, K.J. 2003. The existence value of biodiversity in south Africa: how interest, experience, knowledge, income and perceived level of threat influence local willingness to pay. Ecological Economics 46(2003) 199-216

Velázquez M., R.J. 1998. Estudio financiero para el establecimiento de un módulo de producción pecuaria de doble propósito en el C.E. "La Posta" Tesis de Ingeniero en Desarrollo Agrícola. Universidad Cristóbal Colón. Veracruz, Ver. $69 \mathrm{p}$.

Zhongwei, G., X. Xiao, Y. Gan e Y. Zheng. 2001. Ecosystem functions, services and their values: a case study in Xingshan County of China. Ecological Economics 38:141-154 